\begin{tabular}{|c|c|}
\hline Title & $\begin{array}{l}\text { Therapeutic Strategies for Patients with Internal Carotid or Middle Cerebral A rtery Occlusion Complicated by Severe } \\
\text { Coronary A rtery Disease }\end{array}$ \\
\hline Author(s) & $\begin{array}{l}\text { Kawabori, Masahito; Kuroda, Satoshi; Terasaka, Shunsuke; Nakay ama, Naoki; Matsui, Y oshiro; Kubota, Suguru; } \\
\text { Nakamura, Masanori; Nakanishi, Katsuhiko; Okamoto, Fumiyuki; I wasaki, Y oshinobu }\end{array}$ \\
\hline Citation & $\begin{array}{l}\text { World Neurosurgery, 73(4), 345-350 } \\
\text { https://doi.org/10.1016/.wneu.2010.01.026 }\end{array}$ \\
\hline Issue Date & $2010-04$ \\
\hline Doc URL & http:/hdl.handle.net/2115/70793 \\
\hline Rights & $\begin{array}{l}\text { () 2010. This manuscript version is made available under the CC-BY-NC-ND } 4.0 \text { license } \\
\text { http://creativecommons.org/icenses/by-nc-nd/4.0/ }\end{array}$ \\
\hline Rights(URL) & http://creativecommons.org/icenses/by-nc-nd/4.0/ \\
\hline Type & article (author version) \\
\hline File Information & WorldNeurosurg73_345.pdf \\
\hline
\end{tabular}

Instructions for use 


\section{Therapeutic Strategies for Patients with Internal Carotid or Middle Cerebral Artery Occlusion Complicated by Severe Coronary Artery Disease}

Masahito Kawabori, $\mathrm{MD}^{1}$, Satoshi Kuroda, MD, $\mathrm{PhD}^{1}$, Shunsuke Terasaka, $\mathrm{MD}, \mathrm{PhD}^{1}$, Naoki Nakayama, $\mathrm{MD}, \mathrm{PhD}^{1}$, Yoshiro Matsui, $\mathrm{MD}, \mathrm{PhD}^{2}$, Suguru Kubota, $\mathrm{MD}, \mathrm{PhD}^{2}$, Masanori Nakamura, $\mathrm{MD}, \mathrm{PhD}^{3}$, Katsuhiko Nakanishi, $\mathrm{MD}, \mathrm{PhD}^{4}$, Fumiyuki Okamoto, $\mathrm{MD}, \mathrm{PhD}^{4}$, Yoshinobu Iwasaki, $\mathrm{MD}, \mathrm{PhD}^{1}$

Departments of Neurosurgery ${ }^{1}$, and Cardiovascular Surgery ${ }^{2}$, Hokkaido University Graduate School of Medicine, Sapporo, Japan

Department of Cardiovascular Surgery ${ }^{3}$, Sapporo Medical University, Sapporo, Japan

Department of Cardiovascular Surgery ${ }^{4}$, Teine Keijinkai Hospital, Sapporo, Japan

\section{Correspondence:}

Satoshi Kuroda, MD, PhD,

Department of Neurosurgery, Hokkaido University Graduate School of Medicine, North 15 West 7, Kita-ku, Sapporo 060-8638, Japan

Tel. +81-11-706-5987

Fax. +81-11-708-7737

e-mail: skuroda@med.hokudai.ac.jp 


\section{Abstract}

Objective: Ischemic stroke is one of major complications of cardiac surgery. Although current AHA guideline states that carotid endarterectomy is probably recommended before or concomitant to coronary artery bypass grafting (CABG) for the carotid stenosis, there is no report that analyzed optimal strategies in cardiac surgery for patients with total occlusion of the ICA or MCA. Therefore, this preliminary study was aimed to clarify whether preoperative blood flow measurements and prophylactic STA-MCA anastomosis could reduce the incidence of perioperative ischemic stroke during cardiac surgery in patient with total occlusion of the ICA or MCA.

Methods: This prospective study included 8 patients who were admitted to undergo cardiac surgery including CABG. All of them had total ICA or MCA occlusion on preoperative MR examinations. Preoperative cerebral blood flow and its reactivity to acetazolamide were quantitatively determined in all 8 patients using SPECT or PET.

Results: Preoperative blood flow measurements revealed that 2 (25\%) of 8 patients had normal cerebral hemodynamic because of well-developed collaterals. They safely underwent cardiac surgery. However, a marked impairment of cerebral perfusion reserve was identified in 6 (75\%) of 8 patients in the ipsilateral hemispheres. Of these, 4 patients underwent prophylactic STA-MCA anastomosis prior to CABG. Subsequently, they safely underwent CABG without perioperative ischemic stroke.

Conclusion: This is the first report suggesting that preoperative identification of hemodynamic compromise and prophylactic STA-MCA anastomosis may reduce perioperative ischemic stroke during cardiac surgery in patients with ICA or MCA occlusion, although further studies would be essential to assess the validity.

(242 words)

Key words: coronary artery bypass graft, internal cerebral artery, middle cerebral artery, occlusion, STA-MCA anastomosis, SPECT, PET

Abbreviations: CABG, coronary artery bypass graft; CBF, cerebral blood flow; CVR, cerebrovascular reactivity; ICA, internal carotid artery; ${ }^{123}$ I-IMP, $N$-isopropyl- $p-\left[{ }^{123} \mathrm{I}\right]-$ iodoamphetamine; MCA, middle cerebral artery; MRA, magnetic resonance angiography; PET, positron emission tomography; SPECT, single photon emission computed tomography; STA-MCA, superficial temporal artery to middle cerebral artery 


\section{Introduction}

Ischemic stroke is one of major complications of cardiac surgery, leading to increased mortality and poor functional outcome. Of these, significant stenosis of the internal carotid artery (ICA) stenosis has been accepted as a considerable predictor of poor outcomes in patients undergoing coronary artery bypass graft $(\mathrm{CABG})^{1,2}$. There is increasing evidence that simultaneous or preceding carotid endarterectomy (CEA) is useful in order to reduce the incidence of ischemic stroke in such patients ${ }^{3}$. Carotid artery stenting (CAS) may be an alternative strategy prior to cardiac surgery, although CAS still has the potential risk for perioperative complications, including ischemic stroke and hypotension-induced cardiac failure ${ }^{4}$. Based on the fact that the patients with impaired cerebral perfusion reserve due to ICA or MCA occlusion are at significantly higher risk for subsequent ischemic stroke ${ }^{5}$, the patient with coexisting ICA or MCA occlusion would also be prone to suffer ischemic stroke during cardiac surgery ${ }^{6,7}$. However, there are few systematic studies that denote optimal therapeutic strategy for patients with total occlusion of the ICA or middle cerebral artery (MCA) complicated by coronary artery disease requiring cardiac surgery ${ }^{8,9}$. Very recently, we have demonstrated that preoperative cerebrovascular screening and prophylactic cerebrovascular interventions significantly contribute to reduce the incidence of perioperative stroke in patients with occlusive carotid or vertebral artery diseases ${ }^{1}$.

In this preliminary, prospective study, therefore, we aimed to assess whether preoperative blood flow studies and prophylactic cerebrovascular interventions could safely reduce the incidence of perioperative ischemic stroke during cardiac surgery in patients with ICA or MCA occlusion. Cerebral hemodynamics was evaluated prior to cardiac surgery, using single photon emission computed tomography (SPECT) or positron emission tomography (PET). We believe that continuous accumulation of similar experience would guide us to establish optimal therapeutic strategy in treating these complicated, high-risk patients and to improve their prognosis.

\section{Patients and Methods}

\section{Patients}

This study included 8 patients who were admitted to our hospitals in order to undergo CABG due to refractory coronary artery diseases between April 2002 and September 2007. All of them met the following criteria on preoperative screening tests: (a) total occlusion of the ICA or of the horizontal portion of MCA on MR angiography and (b) no infarction or, if any, small infarction on MRI. Their clinical characteristics are summarized in Table 1 . There were 6 men and 2 women with a mean age of 70.5 years. Preoperative MR angiography revealed total occlusion of the ICA in 4 patients and the MCA in 4 . Of these, three patients have the episode of minor ischemic stroke (modified Rankin scale, 0 to 2) due to ipsilateral occlusive carotid artery diseases. 


\section{Assessment of cerebral hemodynamics}

Cerebral blood flow (CBF) before and 15 minutes after injection of $10-\mathrm{mg} / \mathrm{kg}$ acetazolamide was quantitatively determined, using SPECT and $N$-isopropyl- $p$ - $\left[{ }^{123} \mathrm{I}\right]$-iodoamphetamine (IMP) injection method. Alternatively, the parameter was measured using PET and ${ }^{15}$ O-gas inhalation method ${ }^{10,11}$. To evaluate cerebral perfusion reserve, 10-mm-diameter circular regions of interest (ROIs) were placed in the ipsilateral MCA territories. As described previously, the cerebrovascular reactivity (CVR) to acetazolamide was quantitatively calculated as:

$$
\operatorname{CVR}(\%)=100 \times\left(\mathrm{CBF}_{\mathrm{ACZ}}-\mathrm{CBF}_{\text {rest }}\right) / \mathrm{CBF}_{\text {rest }} \text {, }
$$

where $\mathrm{CBF}_{\text {rest }}$ and $\mathrm{CBF}_{\mathrm{ACZ}}=\mathrm{CBF}$ before and after intravenous injection of acetazolamide, respectively. The values were rated as reduced when any value was less than the mean - 2SD. Based on previous studies, cerebral perfusion reserve was considered as impaired when both CBF and CVR were significantly reduced ${ }^{5,10-12}$.

\section{Treatment protocol}

As shown in Fig. 1, treatment strategies in this study largely depended on the results of preoperative SPECT study and cardiac function in each patient. Thus, CABG was performed without any cerebrovascular intervention when cerebral perfusion reserve was maintained normal because of well-developed collateral circulation. On the other hands, prophylactic superficial temporal artery to middle cerebral artery (STA-MCA) anastomosis was performed prior to CABG when cerebral perfusion reserve was impaired and cardiac function was judged as tolerable to surgery. Both STA-MCA anastomosis and CABG were abandoned and internal medical treatment was continued in patients who had occlusion of multiple cephalic arteries because prophylactic STA-MCA anastomosis on only one side was considered unable to avoid perioperative ischemic stroke during the following cardiac surgery (Fig. 1).

In this study, the frontal and parietal branches of STA were dissected from the scalp under surgical microscope. Then, the Sylvian fissure was widely opened and the anterior and posterior trunks of the MCA were exposed. Subsequently, two branches of STA were anastomosed to the trunks of MCA, respectively. Cardiac surgery such as CABG and off-pump CABG (OPCAB) was performed within 8 weeks after prophylactic STA-MCA anastomosis.

\section{Results}

Overall results

On preoperative SPECT/PET studies, cerebral perfusion reserve was kept normal in 2 of 8 patients included in this study. They underwent OPCAB alone, and their postoperative course was uneventful. Other 6 patients were judged as having impaired cerebral perfusion reserve. Thus, both CBF and CVR were significantly reduced in the ipsilateral MCA territories. Of these 6 
patients, 4 underwent prophylactic STA-MCA anastomosis prior to cardiac surgery. Postoperative course was uneventful. Postoperative blood flow studies revealed significant improvement of cerebral hemodynamics in the operated hemispheres. Subsequently, they safely underwent cardiac surgery, including $\mathrm{CABG}$, OPCAB, and valve replacement. None of them developed perioperative ischemic stroke.

Remaining two patients were judged as having impaired cerebral hemodynamics, but no surgical interventions were applied because they had multiple occlusive arterial diseases (Table 1). Thus, a 67-year-old female had total occlusion of the right MCA and bilateral vertebral artery occlusion. Another 66-year-old male had bilateral ICA occlusion. Their cardiac functions were considered intolerable to multiple cerebrovascular interventions including STA-MCA anastomosis. Therefore, they were medically treated as before. The former died of chronic cardiac failure 4 years later.

\section{Illustrative case (Case 4)}

A 68-year-old man was admitted due to effort angina. He had a past history of acute myocardial infarction when he was 47-years-old, and a minor brain infarction when he was 54-years-old. Cardiac investigation revealed that he had 90\% stenosis at right coronary artery, and also $90 \%$ stenosis at left anterior descending artery with severe mitral regurgitation. Thus, he was thought to be the candidate for CABG and valve replacement. However, preoperative MR angiography and conventional angiography showed total occlusion of the left ICA (Fig. 2A,B). ${ }^{123}$ I-IMP SPECT revealed that both CBF and CVR were significantly reduced in the left MCA territory, suggesting a marked reduction of cerebral perfusion reserve (Fig. 2C,D). First, he underwent left STA-MCA anastomosis to reduce the risk for ischemic stroke during cardiac surgery. Postoperative course was uneventful. MR angiography showed the patency of STA-MCA anastomosis. Follow-up ${ }^{123}$ I-IMP SPECT scans demonstrated significant improvement of cerebral hemodynamics (Fig. 2E,F). He underwent CABG and aortic valve replacement 4 weeks later, and postoperative course was uneventful. He had suffered any coronary nor cerebral ischemic event since then.

\section{Discussion}

\section{Risk of stroke in cardiac surgery}

As aforementioned, ischemic stroke is one of the most devastating complications of cardiac surgery, causing poor functional outcomes and increased mortality, length of hospitalization, and need for long-term care ${ }^{1}$. Especially, the presence of occlusive carotid artery diseases is known to increase the morbidity and mortality in patients undergoing CABG ${ }^{13-16}$. Current AHA guideline recommends carotid endarterectomy (CEA) for both symptomatic and asymptomatic 
patients with severe ICA stenosis. However, there is no convincing guideline about how the patient with ICA or MCA occlusion should be managed when undergoing CABG. Recently, the coincidence of coronary and carotid artery diseases has rapidly been increasing. Recent studies have reported that ICA occlusion can be detected on preoperative examinations prior to CABG in approximately 1.2 to $4 \%$ of patients $6,9,17,18$. The severity of ICA stenosis is closely related to the incidence of perioperative stroke. Thus, Dashe et al. (1987) showed that perioperative stroke occurred in $4.7 \%$ of patients with less than $70 \%$ stenosis, $5 \%$ of patients with $70-80 \%$ stenosis, and $8 \%$ of patients with ICA occlusion ${ }^{7}$. Furthermore, the patients with multiple occlusive carotid diseases are at higher risk for perioperative stroke. Brener et al. (1987) reported that major neurological deficits developed in $14 \%$ of patients with unilateral ICA occlusion, $20 \%$ of patients with significant ICA stenosis and contralateral ICA occlusion, and 33\% of patients with bilateral ICA occlusion ${ }^{6}$. Although the mechanisms that cause ischemic stroke during CABG are multifactorial, hemodynamic insufficiency is considered to account for more than $50 \%$ of its source ${ }^{1,9,16,18}$. Therefore, it is quite important to investigate occlusive carotid artery diseases prior to cardiac surgery and to seek the strategies to reduce the risk of perioperative ischemic stroke in each patient with coronary artery disease.

\section{Assessment of cerebral perfusion reserve}

Especially, we believe that it is essential to maintain cerebral perfusion pressure (CPP) in the cerebral hemisphere ipsilateral to the occluded ICA or MCA. Development of collateral circulation is well known to widely vary among patients with ICA occlusion. Thus, we assessed cerebral hemodynamics in 32 patients with unilateral ICA occlusion, using SPECT and acetazolamide test ${ }^{12}$. Acetazolamide has been widely used to assess cerebral vasodilatory capacity in patients with occlusive carotid artery diseases. All of them could be classified into 4 categories: Type 1 (normal CBF and CVR), Type 2 (normal CBF and reduced CVR), Type 3 (reduced CBF and CVR), and Type 4 (reduced CBF and normal CVR). As the results, 6 (18.8\%) patients were classified as Type 1, 9 (28.2\%) as Type 2, $11(34.4 \%)$ as Type 3 , and $6(18.8 \%)$ as Type $4{ }^{12}$. Previous studies have strongly indicated that CPP is kept within a normal range in Type 1 patients because of well-developed collateral circulation. In Type 2 patients, compensatory vasodilation occurs in response to a moderate reduction in CPP. In Type 3 patients, however, autoregulatory vasodilation could no longer compensate for CPP reduction due to inadequate collateral development, leading to a blood flow decline. In Type 4 patients, a blood flow reduction results from the downregulation of metabolic demand because of ischemia-induced selective neuronal injury ${ }^{11}$. Recent observational studies have shown that the patients with Type 3 ischemia due to ICA or MCA occlusion are significantly at higher risk for subsequent ischemic stroke when they are medically treated ${ }^{5,19}$. Because it is well known that the extraordinary reduction of CPP easily 
induces ischemic attack in the hemodynamically compromised patients ${ }^{20}$, cardiac surgery per se may potentially provoke ischemic stroke in patients with Type 3 ischemia due to ICA or MCA occlusion with or even without cardiopulmonary bypass. Although some anecdotal reports have concluded that CABG can be successfully performed without any cerebrovascular interventions in patients with ICA occlusion ${ }^{8,9}$, it is warranted to establish non-invasive methodology to predict the risk of perioperative ischemic stroke in patients with extracranial and intracranial artery diseases.

\section{Significance of STA-MCA anastomosis prior to cardiac surgery}

Based on these observations, we evaluated the hypothesis that prophylactic STA-MCA anastomosis improves cerebral hemodynamics and reduces the risk for ischemic stroke during cardiac surgery in patients with ICA or MCA occlusion in this preliminary study. As the results, preoperative blood studies revealed that 2 (25\%) of 8 patients had normal CBF and CVR (Type 1) in spite to the ICA or MCA occlusion. They could safely undergo cardiac surgery. On the other hands, other 6 (75\%) of 8 patients had Type 3 ischemia in the MCA territory ipsilateral to the occluded ICA or MCA. Of these, 2 patients had additional occlusive arterial diseases in the contralateral ICA or VA and were medically treated. Remaining 4 patients underwent prophylactic STA-MCA anastomosis prior to cardiac surgery. Subsequently, they safely underwent cardiac surgery including CABG and valve replacement. Interestingly, the incidence of patients with Type 3 ischemia was $75 \%$, being quite high in this study. Previous studies have showed that the prevalence of other occlusive arterial diseases is significantly higher in Type 3 patients than in other patients, suggesting that the development of collateral circulation is inadequate in Type 3 patients ${ }^{12}$. In addition, Type 3 patients are at higher risk for ischemic stroke even in the territories of contralateral carotid and vertebral arteries ${ }^{5,21}$. Therefore, atherosclerosis may more widely be distributed in the cephalic, coronary, and peripheral arteries in patients with Type 3 ischemia. These facts may explain the high incidence of hemodynamic compromise in the patients included in this study.

Impact of STA-MCA anastomosis on long-term outcome in hemodynamically compromised patients has still been undetermined ${ }^{22}$. However, a multi-center randomized trial in Japan have recently shown that STA-MCA anastomosis significantly reduces subsequent incidence of recurrent ischemic stroke in patients with reduced CBF and CVR due to occlusive lesions of the ICA or MCA ${ }^{23}$. In this study, no perioperative ischemic stroke occurred through the combination of preoperative examinations and prophylactic STA-MCA anastomosis. Therefore, preoperative screening may be valuable to identify the patients at higher risk for perioperative ischemic stroke during cardiac surgery, and prophylactic STA-MCA anastomosis may play an important role in preventing it. However, the risk of acute myocardial ischemia and/or cardiac failure during and after STA-MCA anastomosis should always be taken into considerations in treating such patients. 


\section{Limitation of this study}

However, of course, further practice would be necessary to assess its validity, because a case cohort in the present study is too small. In addition, there are no data that asymptomatic hemodynamic compromise due to ICA or MCA occlusion is at high risk for development of cerebral infarction during cardiac surgery. Furthermore, the protocol should be further refined because of the facts that one of two medically treated patients died of chronic cardiac failure 4 years later. As the results, the present study does not provide any conclusive data to support the significance of STA-MCA anastomosis prior to cardiac surgery in patients with ICA or MCA occlusion. However, we believe that the accumulation of such clinical data would be useful to establish the guideline for such patients in the near future.

\section{Conclusion}

This is the first report that suggests that preoperative identification of hemodynamic compromise and prophylactic STA-MCA anastomosis may reduce perioperative ischemic stroke during cardiac surgery in patients with ICA or MCA occlusion, although further studies would be essential to assess the validity. 


\section{References}

1. Nakamura M, Okamoto F, Nakanishi K, et al. Does intensive management of cerebral hemodynamics and atheromatous aorta reduce stroke after coronary artery surgery? Ann Thorac Surg. Feb 2008;85(2):513-519.

2. Suematsu Y, Nakano K, Sasako Y, Kobayashi J, Takamoto S. Strategies for CABG patients with carotid artery disease and perioperative neurological complications. Heart Vessels. 2000;15(3):129-134.

3. Fleisher LA, Beckman JA, Brown KA, et al. ACC/AHA 2007 guidelines on perioperative cardiovascular evaluation and care for noncardiac surgery: a report of the American College of Cardiology/American Heart Association Task Force on Practice Guidelines (Writing Committee to Revise the 2002 Guidelines on Perioperative Cardiovascular Evaluation for Noncardiac Surgery): developed in collaboration with the American Society of Echocardiography, American Society of Nuclear Cardiology, Heart Rhythm Society, Society of Cardiovascular Anesthesiologists, Society for Cardiovascular Angiography and Interventions, Society for Vascular Medicine and Biology, and Society for Vascular Surgery. Circulation. Oct 23 2007;116(17):e418-499.

4. Guzman LA, Costa MA, Angiolillo DJ, et al. A systematic review of outcomes in patients with staged carotid artery stenting and coronary artery bypass graft surgery. Stroke. Feb 2008;39(2):361-365.

5. Kuroda S, Houkin K, Kamiyama H, Mitsumori K, Iwasaki Y, Abe H. Long-term prognosis of medically treated patients with internal carotid or middle cerebral artery occlusion: can acetazolamide test predict it? Stroke. Sep 2001;32(9):2110-2116.

6. Brener BJ, Brief DK, Alpert J, Goldenkranz RJ, Parsonnet V. The risk of stroke in patients with asymptomatic carotid stenosis undergoing cardiac surgery: a follow-up study. J Vasc Surg. Feb 1987;5(2):269-279.

7. Dashe JF, Pessin MS, Murphy RE, Payne DD. Carotid occlusive disease and stroke risk in coronary artery bypass graft surgery. Neurology. Sep 1997;49(3):678-686.

8. Osswald BR, Vahl CF, Fleischer F, Hagl S. Successful revascularisation for unstable angina of a patient with asymptomatic bilateral internal carotid occlusion, 70\% stenoses of the external carotid arteries, and other circulation disturbances. Thorac Cardiovasc Surg. Aug 1997;45(4):200-203.

9. Suematsu Y, Nakano K, Sasako Y, Kobayashi J, Kitamura S, Takamoto S. Conventional coronary artery bypass grafting in patients with total occlusion of the 
internal carotid artery. Heart Vessels. 2000;15(6):256-262.

10. Kuroda S, Shiga T, Houkin K, et al. Cerebral oxygen metabolism and neuronal integrity in patients with impaired vasoreactivity attributable to occlusive carotid artery disease. Stroke. Feb 2006;37(2):393-398.

11. Kuroda S, Shiga T, Ishikawa T, et al. Reduced blood flow and preserved vasoreactivity characterize oxygen hypometabolism due to incomplete infarction in occlusive carotid artery diseases. J Nucl Med. Jun 2004;45(6):943-949.

12. Kuroda S, Kamiyama H, Abe H, Houkin K, Isobe M, Mitsumori K. Acetazolamide test in detecting reduced cerebral perfusion reserve and predicting long-term prognosis in patients with internal carotid artery occlusion. Neurosurgery. Jun 1993;32(6):912-918; discussion 918-919.

13. Breslau PJ, Fell G, Ivey TD, Bailey WW, Miller DW, Strandness DE, Jr. Carotid arterial disease in patients undergoing coronary artery bypass operations. $J$ Thorac Cardiovasc Surg. Nov 1981;82(5):765-767.

14. Hise JH, Nipper ML, Schnitker JC. Stroke associated with coronary artery bypass surgery. AJNR Am J Neuroradiol. Sep-Oct 1991;12(5):811-814.

15. Mickleborough LL, Walker PM, Takagi Y, Ohashi M, Ivanov J, Tamariz M. Risk factors for stroke in patients undergoing coronary artery bypass grafting. $J$ Thorac Cardiovasc Surg. Nov 1996;112(5):1250-1258; discussion 1258-1259.

16. Tunio AM, Hingorani A, Ascher E. The impact of an occluded internal carotid artery on the mortality and morbidity of patients undergoing coronary artery bypass grafting. Am J Surg. Sep 1999;178(3):201-205.

17. Birincioglu L, Arda K, Bardakci H, et al. Carotid disease in patients scheduled for coronary artery bypass: analysis of 678 patients. Angiology. Jan 1999;50(1):9-19.

18. Salasidis GC, Latter DA, Steinmetz OK, Blair JF, Graham AM. Carotid artery duplex scanning in preoperative assessment for coronary artery revascularization: the association between peripheral vascular disease, carotid artery stenosis, and stroke. J Vasc Surg. Jan 1995;21(1):154-160; discussion 161-152.

19. Hokari M, Kuroda S, Shiga T, Nakayama N, Tamaki N, Iwasaki Y. Impact of oxygen extraction fraction on long-term prognosis in patients with reduced blood flow and vasoreactivity because of occlusive carotid artery disease. Surg Neurol. May 292008.

20. Kashiwazaki D, Kuroda S, Terasaka S, Iwasaki Y. Detection of hemodynamic transient ischemic attack during hemodialysis with near-infrared monitoring in a patient with internal carotid artery occlusion. Surg Neurol. Sep 2007;68(3):292-294; discussion 294-295.

21. Ogasawara K, Ogawa A, Yoshimoto T. Cerebrovascular reactivity to acetazolamide 
and outcome in patients with symptomatic internal carotid or middle cerebral artery occlusion: a xenon-133 single-photon emission computed tomography study. Stroke. Jul 2002;33(7):1857-1862.

22. Vilela MD, Newell DW. Superficial temporal artery to middle cerebral artery bypass: past, present, and future. Neurosurg Focus. 2008;24(2):E2.

23. Ogasawara K, Ogawa A. [JET study (Japanese EC-IC Bypass Trial)]. Nippon Rinsho. Oct 28 2006;64 Suppl 7:524-527. 


\section{Figure Legends}

\section{Fig. 1}

Present treatment scheme for patients with total occlusion of the internal carotid artery or middle cerebral artery complicated by coronary artery disease requiring cardiac surgery.

ICA, internal carotid artery; MCA, middle cerebral artery; CBF, cerebral blood flow; CVR, cerebrovascular reactivity; STA-MCA, superficial temporal artery to middle cerebral artery

\section{Fig. 2}

Pre- and post-operative radiological findings of a 68-year-old man who was diagnosed as angina pectoris and mitral regurgitation. Pre-operative MR angiography showed total occlusion of the left internal carotid artery (ICA, A). Conventional angiography revealed that a little collateral circulation can be seen from the right ICA to the left middle cerebral artery (MCA) territory through the anterior communicating artery (B). Pre-operative ${ }^{123}$ I-IMP SPECT revealed significant reduction of cerebral blood flow at resting state (C) and its reactivity to acetazolamide (ACZ, D) in the left MCA territory. Postoperative SPECT showed the improvement of cerebral circulation (rest: E, ACZ: F). 


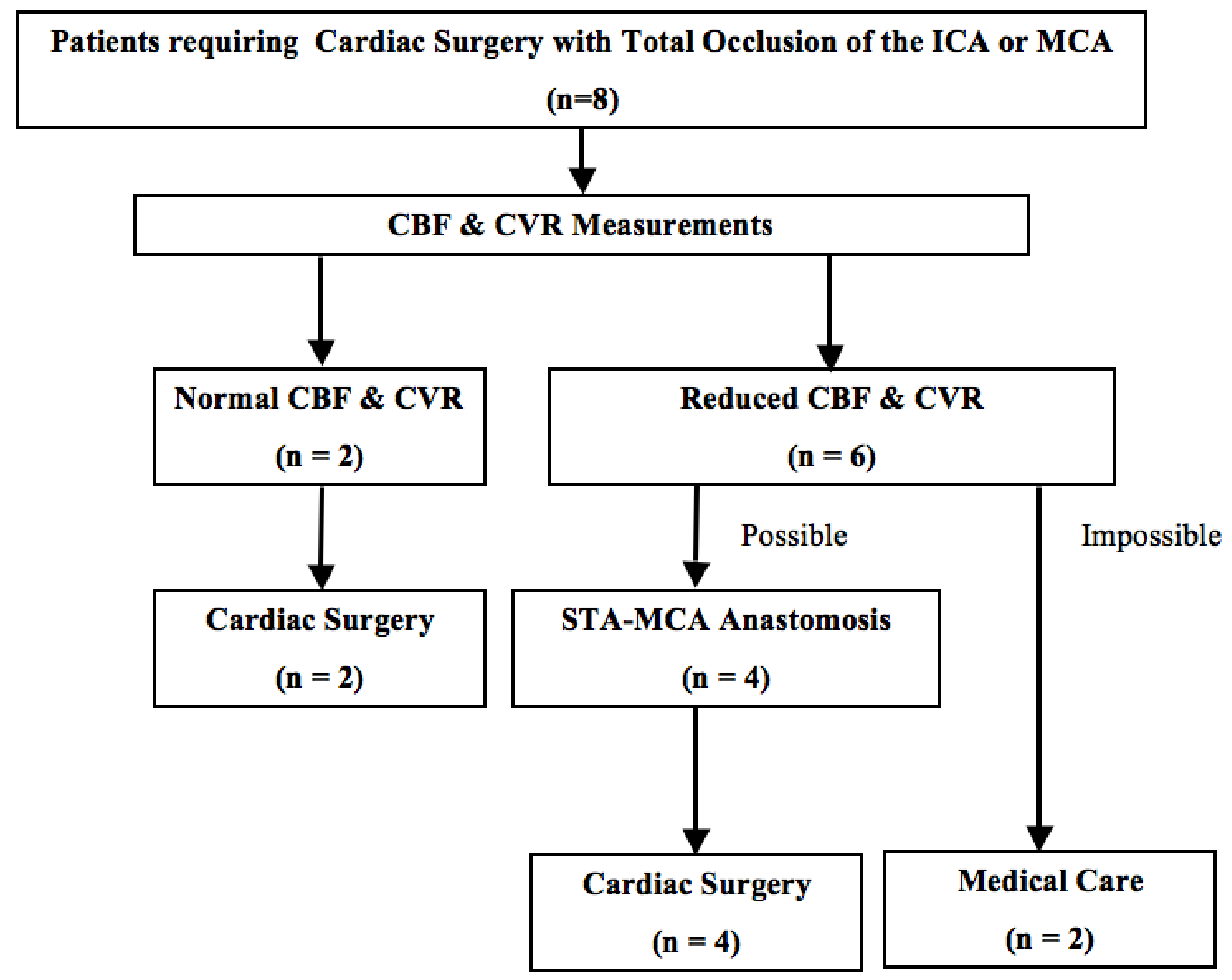



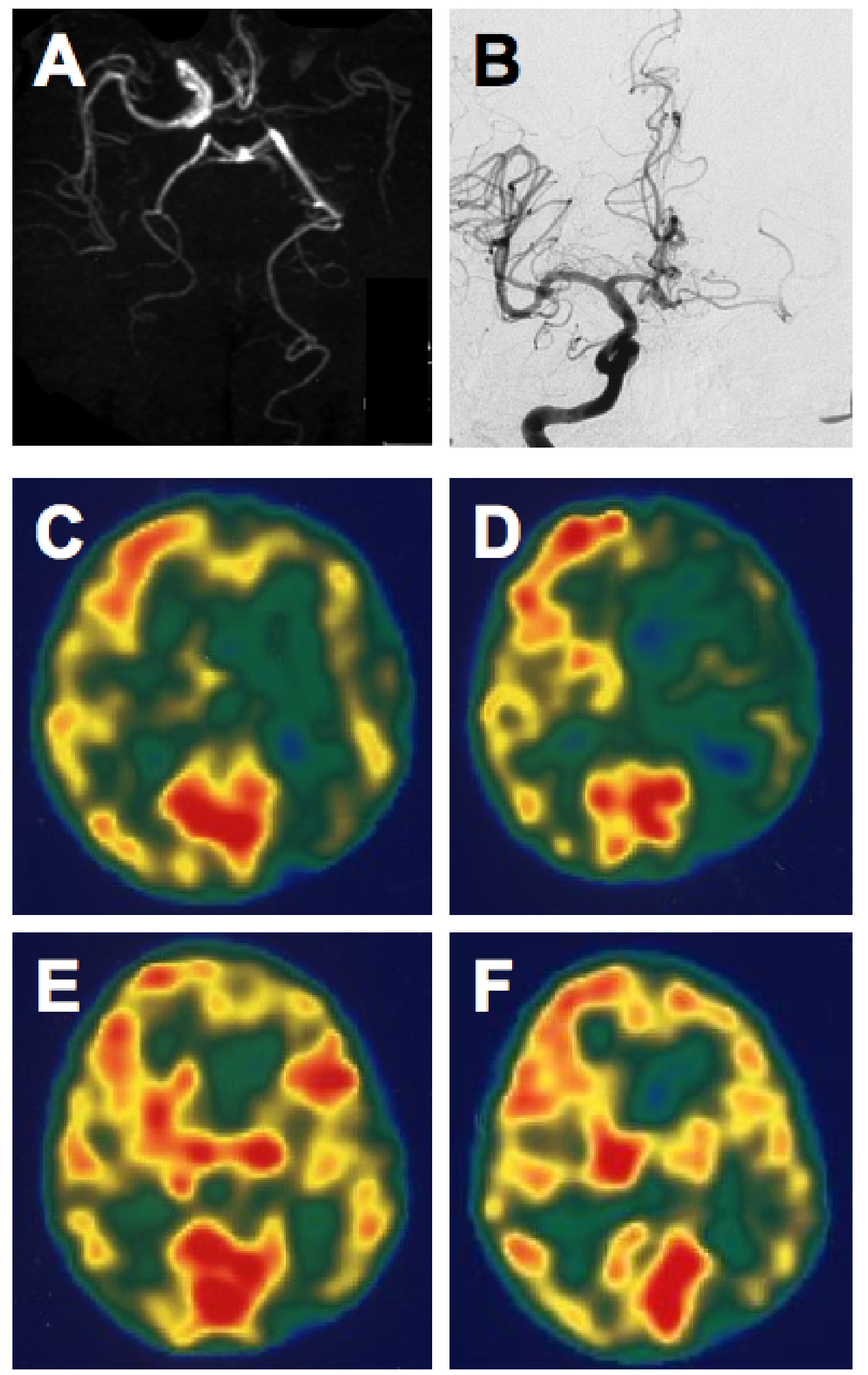

Fig. 2 
Summary of clinical information and results

\begin{tabular}{|c|c|c|c|c|c|c|c|c|c|c|c|}
\hline case & Age/Sex & risk factor & $\begin{array}{c}\text { ischemic heart } \\
\text { disease }\end{array}$ & coronary artery disease & CVD & craniocervical lesions & $\begin{array}{l}\text { baseline } \\
\text { CBF }\end{array}$ & CVR & OEF & treatment strategy & outcome \\
\hline 1 & $70 / \mathrm{M}$ & HT, smoking & EAP & RCA(99\%), LAD(75\%), LCX(100\%) & asymptomatic & It ICA occlusion & $\rightarrow$ & $\rightarrow$ & - & OPCAB alone & no deficit \\
\hline 2 & $81 / \mathrm{M}$ & $H T, D M$ & EAP & RCA $(99 \%)$, LAD $(90 \%)$ & asymptomatic & It MCA occlusion & $\rightarrow$ & $\rightarrow$ & - & OPCAB alone & no deficit \\
\hline 3 & $70 / \mathrm{M}$ & HT, DM & EAP & RCA $(99 \%), \operatorname{LAD}(90 \%), \operatorname{LCX}(90 \%)$, AS & asymptomatic & rt MCA occlusion & $\downarrow$ & $\downarrow$ & $\uparrow$ & STA-MCA bypass $\rightarrow$ CABG $、$ AVR & $\begin{array}{l}\text { acute heart failure after } \\
\text { STA-MCA bypass }\end{array}$ \\
\hline 4 & $68 / \mathrm{M}$ & $\mathrm{HT}, \mathrm{CHF}$ & EAP, OMI, MR & RCA(90\%), LAD(75\%), MR & OCI & It ICA occlusion & $\downarrow$ & $\downarrow$ & - & STA-MCA bypass $\rightarrow$ CABG, MVR & no deficit \\
\hline 5 & $75 / F$ & HT & EAP & $\operatorname{RCA}(100 \%), \operatorname{LAD}(90 \%), \operatorname{LCX}(100 \%)$ & OCI & rt MCA occlusion & $\downarrow$ & $\downarrow$ & $\rightarrow$ & STA-MCA bypass $\rightarrow$ CABG & no deficit \\
\hline 6 & $67 / M$ & $\mathrm{HT}, \mathrm{HL}$ & UAP & RCA(90\%), LAD(90\%), LCX(75\%) & asymptomatic & rt ICA occlusion & $\downarrow$ & $\downarrow$ & - & STA-MCA bypass $\rightarrow$ OPCAB & AP after STA-MCA bypass \\
\hline 7 & $67 / F$ & HT, DM & AHF, MR & RCA $(90 \%), \operatorname{LCX}(75 \%)$ & OCI & $\begin{array}{l}\text { rt MCA occlusion, bil } \\
\text { VA occlusion }\end{array}$ & $\downarrow$ & $\downarrow$ & $\uparrow$ & medication & no deficit \\
\hline 8 & $66 / \mathrm{M}$ & HT, DM & AMI & $\operatorname{LAD}(99 \%), \operatorname{LCX}(99 \%)$ & asymptomatic & bil IC occlusion & $\downarrow$ & $\downarrow$ & - & medication & no deficit \\
\hline
\end{tabular}



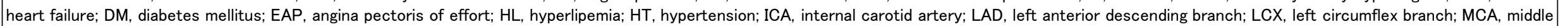


STA-MCA, superficial temporal artery to middle cerebral artery; UAP, unstable angina pectoris; VA, vertebral artery; -, not examined; (\%), stenosis 